\title{
Design, Simulation and Analysis of NEMS based Piezoresistive Pressure Sensor
}

\author{
Ayyappa Vajjaramatti \\ Department of Electronics and Communication Engineering \\ Basaveshwar Engineering College (A), \\ Baglkot-587103, India
}

\author{
Kirankumar B. Balavalad \\ Department of Electronics and Communication Engineering \\ Basaveshwar Engineering College (A), \\ Baglkot-587103, India
}

\author{
Ashokkumar M \\ Department of Electronics and Communication Engineering \\ Basaveshwar Engineering College (A), \\ Baglkot-587103, India
}

\begin{abstract}
In this work, we present the design, simulation and analysis of NEMS based piezoresistive nano pressure sensor operating over a pressure range of 0 to $0.5 \mathrm{MPa}$. The entire sensor dimension is in nano scale. Nano piezoresistors are placed and are connected in the form of Wheatstone Bridge, on the diaphragm. The sensor is simulated for the mechanical and electrical performance parameters. The designed sensor has a sensitivity of $1.28 \mu \mathrm{V} / \mathrm{Pa}$, which is better at nano scale. Such kind of pressure sensors are best suited for medical pressure sensing applications.
\end{abstract}

Keywords-NEMS, Nano piezoresistive pressure sensors, sensitivity.

\section{INTRODUCTION}

Nanotechnology has paved way for many innovative devices that can bring about the change in the current sensing and actuation technology. Nanotechnology can develop sensors with enhanced sensitivity and quick response. Microelectronics technology is now slowly moving towards nanoelectronics technology. Currently MEMS (Micro Electro-Mechanical Systems) technology is used for development of sensors and actuators, where the scale happens to be in microns. NEMS (Nano Electro-Mechanical Systems) is an extension of MEMS, here the scale is in nano. Large number of sensors have been developed using MEMS technology, but using Nanotechnology the sensors are expected to perform better than as they use to in MEMS. In the work that we demonstrate in this paper is design and development of nano piezoresistive pressure sensor. With the intent of making devices small, researchers have started using NEMS as an alternative to MEMS technology. Although fabrication of NANO structures might not be as simple as it is for MEMS, but nano devices could perform better then MEMS devices. Piezoresistive pressure sensors in its basic form will contain a thin diaphragm over which four piezoresistors are placed and are connected in the form of Wheatstone bridge. When pressure is applied on diaphragm it will displace and transfer the applied stress on the piezoresistors which in turn convert the applied stress into readable electrical potential. In the works presented in [1]-[3] have designed and analyzed MEMS based piezoresistive pressure sensors and have drawn lot of facts about these devices such as 1 . Square diaphragm is best suited for design of piezoresistive pressure sensor. 2 . Piezoresistors must be placed at the high stress regions, 3 .
Piezoresistors must be selected with high gauge factor, 4. these sensors should be back pressurized.

Papers [4]-[6] have worked on deign and analysis of piezoresistive pressure sensors. They have clearly shown the design aspects of piezoresistive pressure sensors. Many works have been carried out to enhance the sensitivity of these sensors, few to be noted are [7]-[9], were the authors have used SOI, CNT and Silicon nano wire technologies [10], [11]. Although the fabrication aspects of nano piezoresistive pressure sensors re not easy, int his paper we have made an attempt to design and FEM based analysis nano piezoresistive pressure sensor over a pressure range of $0.5 \mathrm{MPa}$. The idea to design and analyze the nano sensor is that, this nano sensor provides better sensitivity compared to micro counterparts and also can sense very low mount of analytes too, when used for bio-sensing applications. The sensor is designed using silicon and doped silicon variants. The material properties and dimensions used re mentioned in the subsequent sections. The sensor design has sensitivity of $\mathrm{nm} / \mathrm{MPa}$

\section{DESIGN OF PIEZORESISTIVE NANO PRESSURE SENSOR}

Typical piezoresistive pressure sensor (PPS) consists of a diaphragm most of the time made up of silicon, on top of which four nano Piezoresistors (usually formed by controlled doping of silicon) are either embedded or (deposited). Then each of the piezoresistors are connected in the form of Wheatstone bridge. The simple diagram is shown in fig. 1. For the configuration shown in fig. 1, pressure is applied on the diaphragm, which will deflect. Four nano piezoresistors will experience this stress and undergo change in the resistance. Which will be further sensed by the bridge. Diaphragm dimensions, size and Piezoresistors dimensions, shape and placement are very important design considerations. Burst pressure, sensitivity and linearity are important performance parameters of the sensors. The sensor dimensions are mentioned in Table. 1. The whole device dimension itself is $700 \mathrm{~nm}$ x $700 \mathrm{~nm}$ (including the diaphragm). And the material properties used are mentioned in table. 2. Fig. 1. Is also a model used for simulation study. 


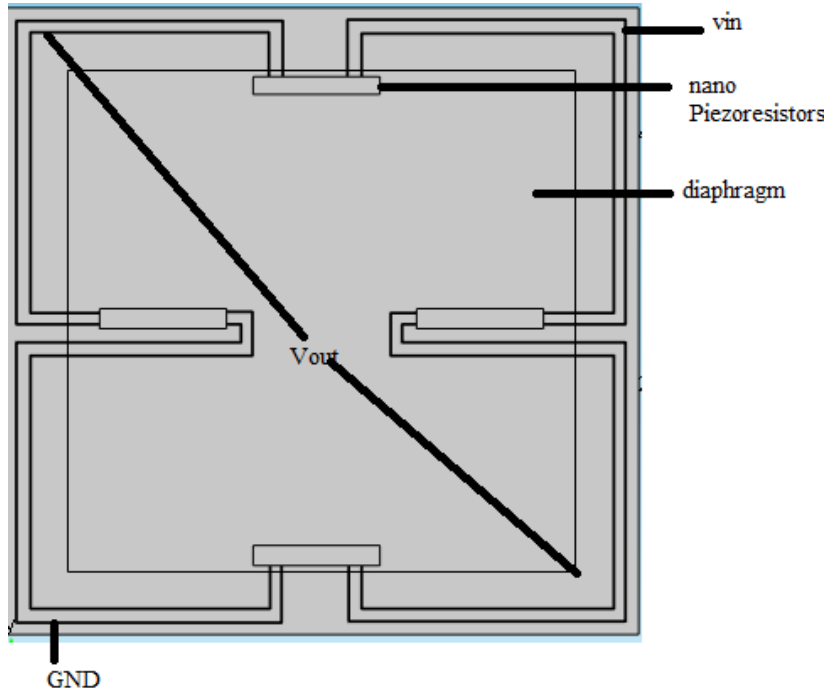

Fig.1. NEMS based Piezoresistive Pressure Sensor simulation model

In the above Fig. 1., piezoresistors are placed in such a way that 2 of them will experience maximum change in resistance and two will face least change. By the virtue of this the bridge gets unbalanced and will provide better output voltage. A known pressure ranging from 0 to $0.5 \mathrm{MPa}$ id applied to the diaphragm. Corresponding displacement and potential analysis are done and are mentioned in detail in results section. The mechanical characterization of the sensor is discussed in simulation section. Chrome + gold have been used for connecting the piezoresistors and to form Wheatstone Bridge.

TABLE 1. Sensor dimensions

\begin{tabular}{|c|c|c|c|}
\hline Sl. No & Mechanical Structure & Material & Dimension $(\mathrm{nm})$ \\
\hline 1. & Diaphragm & n-type Silicon & $500 \times 500 \times 1$ \\
\hline 2. & Piezoresistors & p-type silicon & $140 \times 10 \times 5$ \\
\hline 3. & Connectors & Chrome-gold & $\begin{array}{c}\text { Through out the } \\
\text { diaphragm for } \\
\text { connection }\end{array}$ \\
\hline
\end{tabular}

TABLE 2. Material properties

\begin{tabular}{|c|c|c|c|c|c|}
\hline $\begin{array}{c}\text { Sl. } \\
\text { No }\end{array}$ & Material & $\begin{array}{c}\text { Density } \\
\left(\mathrm{kg} / \mathrm{m}^{3}\right)\end{array}$ & $\begin{array}{c}\text { Young's } \\
\text { modulus } \\
(\mathrm{GPa})\end{array}$ & $\begin{array}{c}\text { Poission's } \\
\text { ratio }\end{array}$ & $\begin{array}{c}\text { Piezoresistive } \\
\text { coefficient } \\
\left(\mathrm{Pa}^{-1}\right)\end{array}$ \\
\hline 1. & Silicon & 2330 & 170 & 0.23 & $30 \times 10^{-11}$ \\
\hline 2. & $\begin{array}{c}\text { n-type } \\
\text { silicon }\end{array}$ & 2270 & 70.0 & 0.23 & $78 \times 10^{-11}$ \\
\hline 3. & $\begin{array}{c}\text { p-type } \\
\text { silicon }\end{array}$ & 2270 & 70.0 & 0.23 & $138 \times 10^{-11}$ \\
\hline
\end{tabular}

\section{SIMULATION OF NANO PIEZORESISTIVE PRESSURE SENSOR}

The simulation model of the sensor is shown in Fig. 2. The simulation comprises of diaphragm, four piezoresistors placed on top of the diaphragm and connectors connecting the resistors.

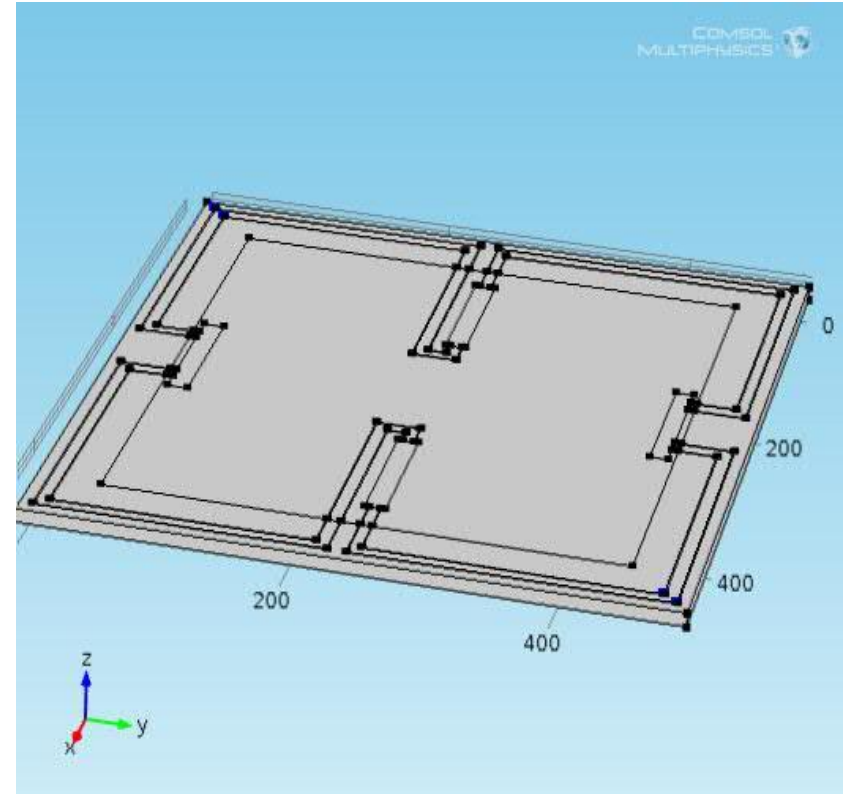

Fig. 2. Simulation Model of Nano Piezoresistive Pressure Sensor

The sensor is further subjected to FEM analysis. The parameters used for mechanical characterization are mentioned below.

1. Burst pressure: Burst pressure is very important design factor for the sensors. Burst pressure actually decides the maximum pressure that can be applied to the diaphragm. When pressure is applied to the diaphragm, the stress on the diaphragm will increase. When the maximum stress on the diaphragm exceeds the yield strength of the diaphragm material, then the diaphragm will burst and undergo plastic deformation. Burst pressure of the sensor is calculates as using equation 1.

$P_{\text {burst }}=\frac{3.4 \sigma_{\max } h^{2}}{\left(1-v^{2}\right) A}$

Where, A-area of the diaphragm, v-poisons ratio, hheight of the diaphragm, $\sigma$-fracture stress on the diaphragm. The calculated burst pressure is around $11 \mathrm{MPa}$ for the sensor. Hence pressure applied for analysis is $0.5 \mathrm{MPa}$ the deflection that pressure will reach/exceed $1 / 5^{\text {th }}$ of the diaphragm max deflection.

2. Maximum deflection:

Maximum centre deflection calculation varies

3. depending upon the type of the diaphragm. The maximum center deflection for a square diaphragm is expressed in equation $3 \& 4$.

$$
\mathrm{W}_{\text {max }}=0.01512 \frac{\mathrm{Pa}^{3}}{\mathrm{D}}
$$

Where, $\mathrm{P}$ in equation (3.2) is pressure applied, ' $\mathrm{a}$ ' is the side of the square diaphragm and ' $D$ ' is the flexural density expressed in equation (3.3) as,

$$
D=\frac{E h^{3}}{12\left(1-v^{2}\right)}
$$

Where, ' $E$ ' is the young's modulus, ' $h$ ' is the height of

1. the diaphragm and ' $v$ ' is the poisons ratio. Maximum deflection for the sensor designed at $1 \mathrm{MPa}$ pressure is $2.32 \mathrm{~nm}$ 
In the next section results and the electrical characterizations of the sensor is discussed.

\section{RESULTS AND ELECTRICAL CHERECTERIZATION OF THE SENSOR}

The above mentioned sensor is subjected to FEM based mechanical and electrical analysis. For both the analysis the input pressure is varied from 0 to $0.5 \mathrm{MPa}$ and corresponding displacement of the diaphragm and output voltage of the bridge is observed. Further sensitivity of the sensor is calculated and validated. Fig. 3 presents the displacement plot of the diaphragm at $0.5 \mathrm{MPa}$ of the input pressure applied. Fig. 4. Shows the simulated image of the all four piezoresistors on top of diaphragm at an applied pressure of $0.5 \mathrm{MPa}$. one can clearly observe two resistors have displaced lot compared to the other two. The displacement (nm) v/s applied pressure (MPa) graph for the sensor diaphragm is shown in Fig. 5. The plot shows both theoretical and simulated values of the sensor. The sensor is subjected to the variable input known pressure of 0 to $0.5 \mathrm{MPa}$ on the diaphragm.

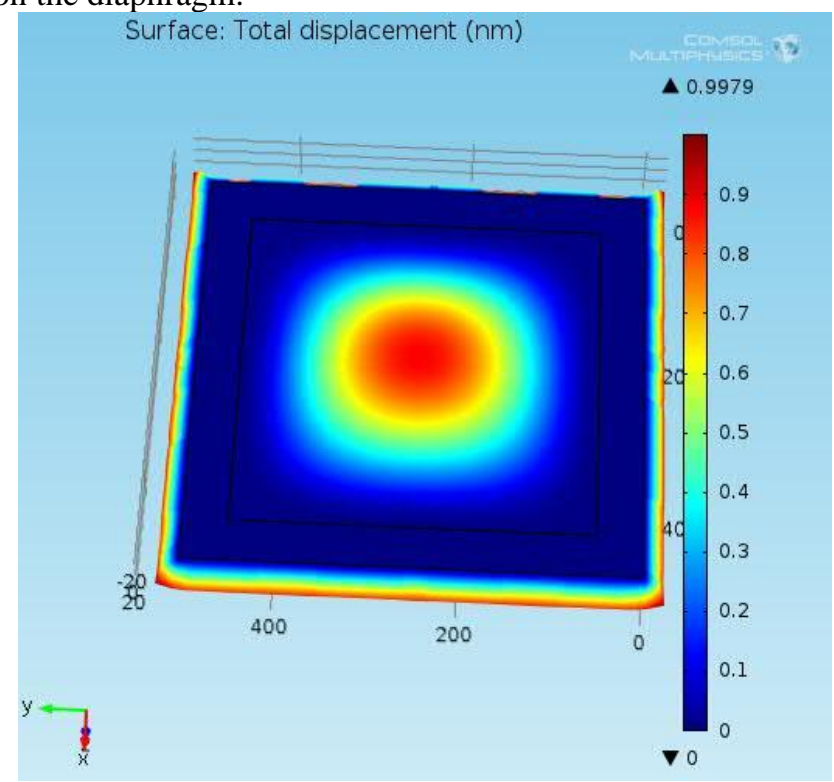

Fig. 3. Displacement of diaphragm at $0.5 \mathrm{MPa}$ pressure

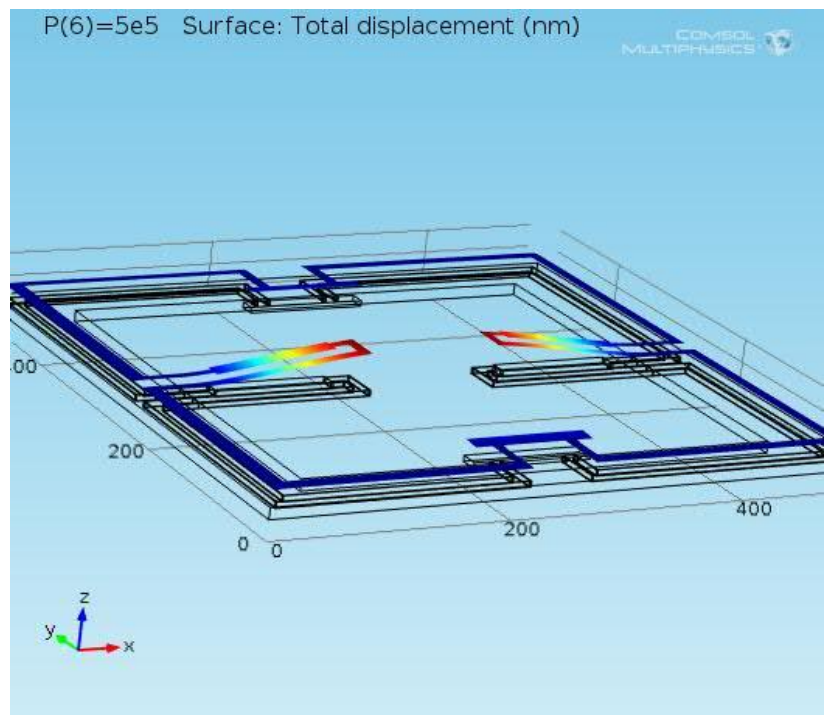

Fig. 4. Displacement all four piezoresistors at $0.5 \mathrm{MPa}$ pressure
The maximum von misses stress distribution on the sensor due to applied pressure at $0.5 \mathrm{MPa}$ is $2.4 \times 10^{9} \mathrm{~Pa}$.

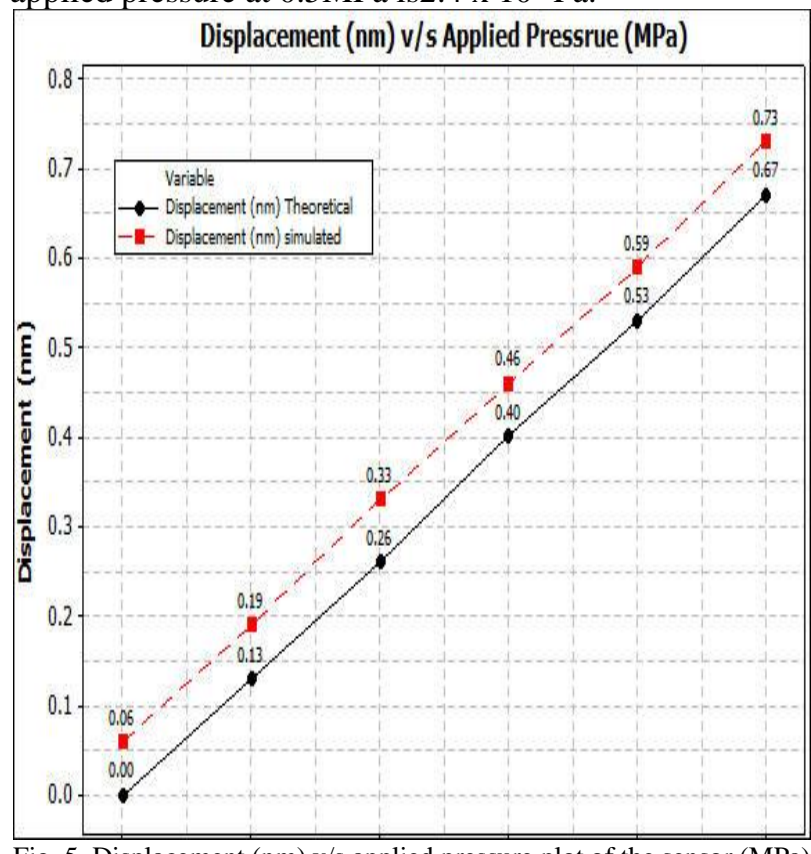

Fig. 5. Displacement (nm) v/s applied pressure plot of the sensor (MPa)

\section{A. Electrical Characterization}

The sensor is subjected to electrical characterization for sensor parameters such as output change in resistivity, output voltage in response to applied input pressure and sensitivity. The electrical characterization is mainly concerned with the piezoresistors, initially the sheet resistivity of the material selected for resistors design in $78 \times 10^{-3} \Omega \mathrm{m}$. Using equation 3 , the initial resistance of the resistors is calculated as $7.8 \times 10^{-3}$ $\Omega$.

$$
R=\frac{\rho L}{A}
$$

Where, $\rho$-resistivity, L-length of the resistors and A-area of the resistors. The bridge needs input bias voltage, we have considered it to be $1.6 \mathrm{~V}$, so that a power from a small battery can be used to provide necessary bias. The $\Delta R / R$ value of the sensor for applied pressure at $1.6 \mathrm{~V}$ constant bias potential is presented in graph shown in Fig. 6. The values of $\Delta R / R$ at different input pressure is mentioned on the graph itself.

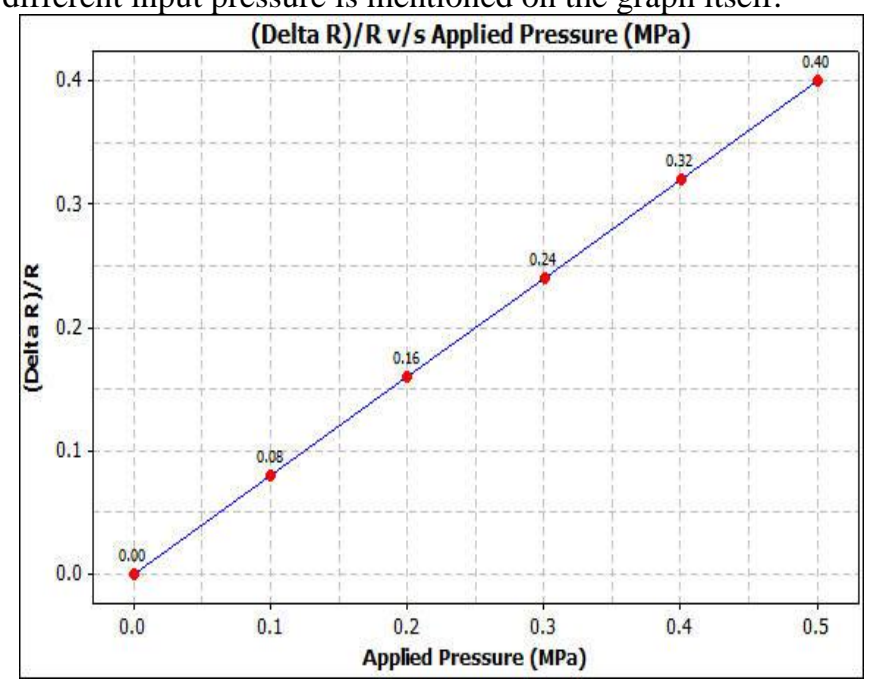

Fig. 6. (Delta R)/R v/s Applied Pressure (MPa) 
Further the output voltage of the bridge containing resistors have been analyzed for an input constant bias voltage of $1.6 \mathrm{~V}$. The plot of the same containing with theoretical and simulated data points has been presented in Fig. 7. And the equation used to calculate the output potential of the bridge is expressed as below in equation 5 .

$$
V_{\text {out }}=P \frac{a^{2}}{h^{2}}(1-v) \pi_{44} V_{\text {in }}
$$

Where, $\mathrm{P}-$ Pressure applied ( 0 to $0.5 \mathrm{MPa})$, a - area of the diaphragm, $\mathrm{h}-$ height/thickness of the diaphragm, $\mathrm{v}-\mathrm{v}$ Poisson's ratio, $\pi_{44^{-}}$piezoresistive coefficient $\left(138 \times 10{ }^{11} \mathrm{~Pa}^{-}\right.$ ${ }^{1}$ ) of the resistor material and $V_{\text {in }}$ - input bias voltage (1.6V).

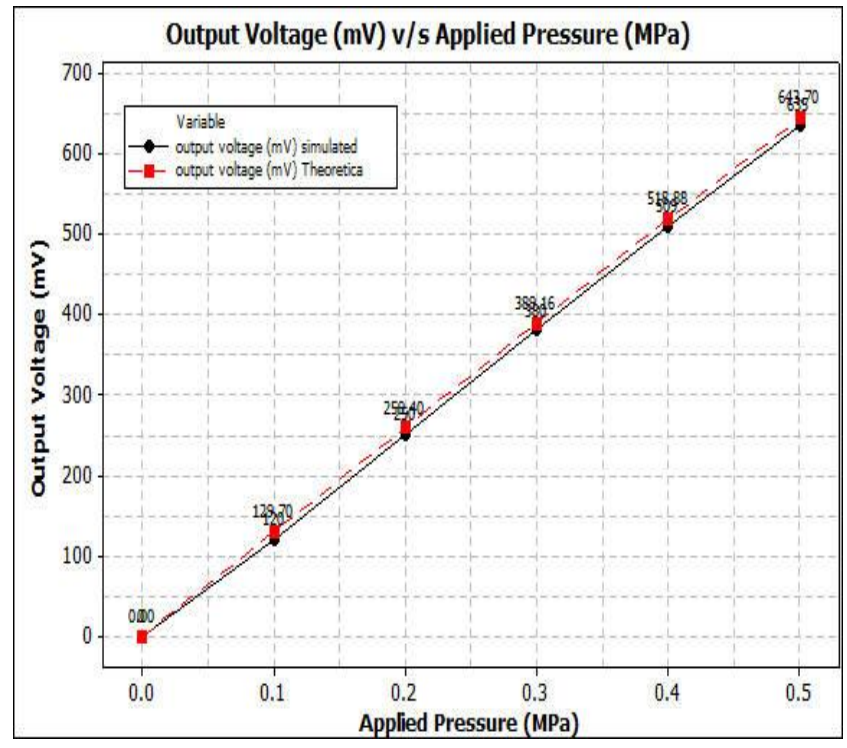

Fig. 7. Output voltage (mV) v/s Applied Pressure (MPa)

B. Sensitivity calculation

The sensitivity of the sensor is calculated as shown in equation 6. With the unit $\mathrm{mV} / \mathrm{MPa}$. Sensitivity can also be calculated as mentioned in equation 7 .

$$
\begin{aligned}
& S=\frac{\Delta V}{\Delta P} \\
& S=\frac{\Delta V}{\Delta P} \frac{1}{V_{\text {in }}}
\end{aligned}
$$

The calculated sensitivity at pressure samples of $0.5 \mathrm{MPa}$ and $0.1 \mathrm{MPa}$ turns out to be $1.28 \mu \mathrm{V} / \mathrm{Pa}$. the simulated sensitivity and theoretical sensitivity are mentioned in Table. 3. The simulated and theoretical sensitivity values of the proposed sensor match each other.

\section{CONCLUSION}

The paper discusses the design, simulation and analysis of a NEMS based piezoresistive pressure sensor operating at a pressure range of 0 to $0.5 \mathrm{MPa}$. All dimensions of the sensor is in nano scale The sensor designed has a better sensitivity of 1.28 $\mu \mathrm{V} / \mathrm{Pa}$ and has a potential applications in the medical field. These kind of nano pressure sensors can be used in medical health care applications to measure various pressures in human body. As the entire sensor size is in nano scale, this sensor can be thought of as a invasive sensor for pressure monitoring in humans.

\section{REFERENCES}

[1] K. N. Bhat and M. M. Nayak, "MEMS Pressure Sensors- An Overview of Challenges in Technology and Packaging," Inst. Smart Struct. Syst. J. Isss J. Isss, vol. 1, no. 1, pp. 1-10, 2012.

[2] V. Balaji and K. N. Bhat, "A Comparison of Burst Strength and Linearity of Pressure Sensors having Thin Diaphragms of Different Shapes," J. Isss, vol. 2, no. 2, pp. 18-26, 2013, [Online]. Available: http://www.isssonline.in/journal-isss/journalarchives.html\#223.

[3] S. Santosh Kumar, A. K. Ojha, R. Nambisan, A. K. Sharma, and B. D. Pant, "Design and Simulation of MEMS Silicon Piezoresistive Pressure Sensor for Barometric Applications," Int. Conf. Adv. Recent Technol. Electr. Electron., pp. 339-345, 2013

[4] K. B. Balavalad and B. G. Sheeparamatti, "Design simulation and analysis of piezoresistive micro pressure sensor for pressure range of 0 to 1MPa," 2017, doi: 10.1109/ICEECCOT.2016.7955243.

[5] S. Meti, K. B. Balavalad, and B. G. Sheeparmatti, "Sensitivity Analysis of Silicon Based Micro Pressure Sensors Using Different Configurations of Meander Shape Piezoresistors," vol. 6, no. 5, pp. 6241-6245, 2016, doi: 10.4010/2016.1506.

[6] S. Bannikoppa, A. C. Katageri, K. B. Balavalad, and B. G. Sheeparamatti, "Design of Piezoresistive pressure sensor for enhanced sensitivity," 2016 Int. Conf. Energy Effic. Technol. Sustain. ICEETS 2016, pp. 706-710, 2016, doi: 10.1109/ICEETS.2016.7583841.

[7] B. G. Sheeparamatti and K. B. Balavalad, "Fabrication and characterization of polysilicon-on-insulator (PolySOI) and a-SOI based micro piezoresistive pressure sensor for harsh environment applications," Microsyst. Technol., vol. 25, no. 11, pp. 4119-4133, 2019, doi: 10.1007/s00542-019-04358-7.

[8] K. B. Balavalad and B. G. Sheeparamatti, "Design, Simulation Analysis of $\mathrm{Si}$, SOI Carbon Nanotube (CNT) based Micro Piezoresistive Pressure Sensor for a High Tmeperature Pressure," 2018, doi: 10.1109/ICCSDET.2018.8821104.

[9] K. B. Balavalad and B. G. Sheeparamatti, "Design, simulation analysis of SOI based micro piezoresistive pressure sensor for high temperature applications," 2018, doi: 10.1109/RTEICT42901.2018.9012626.

[10] P. Song et al., "A novel piezoresistive MEMS pressure sensors based on temporary bonding technology," Sensors (Switzerland), vol. 20, no. 2, pp. 1-10, 2020, doi: 10.3390/s20020337.

[11] L. Lou et al., "Characteristics of NEMS piezoresistive silicon nanowires pressure sensors with various diaphragm layers," Procedia Eng., vol. 25, no. December, pp. 1433-1436, 2011, doi: 10.1016/j.proeng.2011.12.354.

Table. 5. Sensitivity values of the sensor

\begin{tabular}{|c|c|}
\hline \multicolumn{2}{|c|}{ Sensitivity of the sensor $(\boldsymbol{\mu V} / \mathbf{P a})$} \\
\hline Theoretical & Simulated \\
\hline 1.28 & 1.28 \\
\hline
\end{tabular}

